Cockcroft 07-26

\title{
SIMULATIONS OF STRETCHED WIRE MEASUREMENTS OF 3.9GHZ CAVITIES FOR THE ILC
}

B.F. Spencer, N. Chanlek, R. M. Jones, Cockcroft Institute /University of Manchester, UK

G. Burt, Cockcroft Institute/Lancaster University, UK

P. Goudket, Cockcroft Institute /ASTeC, Daresbury Laboratory, UK 


\title{
SIMULATIONS OF STRETCHED WIRE MEASUREMENTS OF 3.9GHZ CAVITIES FOR THE ILC
}

\author{
B.F. Spencer, N. Chanlek, R. M. Jones, Cockcroft Institute/University of Manchester, UK \\ G. Burt, Cockcroft Institute/Lancaster University, UK \\ P. Goudket, Cockcroft Institute /ASTeC, Daresbury Laboratory, UK
}

\begin{abstract}
We present simulations on the transverse modes in crab cavities required in the beam delivery system of the ILC to maximise the luminosity of the beam. We utilize both finite difference and finite element computer codes to simulate the electromagnetic fields in these cavities in the presence of a stretched wire. This study is intended to both predict the wake-field in experiments on the modal characterisation of $3.9 \mathrm{GHz}$ cavities in progress at the Cockcroft Institute. A scattering matrix approach is used on multi-cell cavities and compared with experimental data. Results are presented on the modes in crab cavity structures subjected to infinitely periodic boundary conditions.
\end{abstract}

\section{INTRODUCTION}

In order to achieve acceptable luminosity for the ILC, $\mathrm{RF}$ deflecting cavities are required to rotate the electron and positron bunches before they reach interaction point (IP). The present design incorporates a $14 \mathrm{~m} . \mathrm{rad}$ crossing angle and without the crab cavities there would be a $30 \%$ loss in luminosity [1]. These deflecting "crab" cavities utilise the first dipole mode, operate in a $\pi$ mode phase advance per cell, and at a frequency of $3.9 \mathrm{GHz}[2,3]$.

There is a need to verify the cavity design with a bench top measurement. Furthermore, when fabricating several of these structures it will also be necessary to test cavities during manufacture to ensure that fabrication errors have not led to a degradation of the crabbing mode and have not significantly re-distributed the lower order monopole modes and higher order dipole modes. One proposed method to characterise the modes, is the stretched wire measurement of impedance [4], originally proposed by Sands [5] and successfully used on X-band structures for the NLC [6]. The fundamental idea behind replacing a beam with a wire is grounded in a comparison of the physics of electromagnetic waves excited in both situations. An ultra-relativistic beam in free space radiates electromagnetic waves that are essentially entirely transverse to the direction of propagation and the field excited on a coaxial wire is also TEM. Thus, we 'simulate' the transverse electromagnetic field excited by a charged particle beam with a TEM wave excited on a wire stretched within a cavity. The field scattered from discontinuities represents the wake-field excited by the particle beam.

We have fabricated several inner and end cells of a proposed 9-cell crab cavity. This structure is modular in design, in that a single or several cells (up to 9) can be bolted together and measured [7].
In order to provide verification on the accuracy of the stretched wire technique we made measurements on the scattering matrix properties of a 3-cell crab cavity. As we restricted the number of cells in this experiment, we were able to make an accurate prediction of the scattering matrix and impedance properties using a finite element computer code known as High Frequency Structure Simulator (HFSS) v8.5 [8]. The three cells used in this study were one middle cell of the crab cavity and two ends cells.

We also performed simulations based on the middle cell subjected to infinitely periodic boundary conditions in order to study the properties of the higher order modes (HOMs). For this simulation we utilised the finite difference code GdfidL [9].

This paper is organised as follows. The following section is focussed on a study of electromagnetic fields in the cavity and the influence of the wire on the modes excited by a wire inserted longitudinally within the cavity and offset transversely. These results are compared with experimental data. In the penultimate main section, we analyse the HOMs and present them in the form of a Brillioun diagram.

\section{IMPEDANCE OF A 3-CELL CAVITY}

The wake-field excited by an energetic charged particle beam is conveniently represented in terms of its Fourier transform, the impedance. As a preliminary step to computing the impedance, it is necessary to evaluate the transmission component of the scattering matrix. We utilised HFSS in the driven mode for these simulations. The mesh used to model the wire-loaded cavity at the $\pi$ mode frequency is illustrated in Fig 1. In

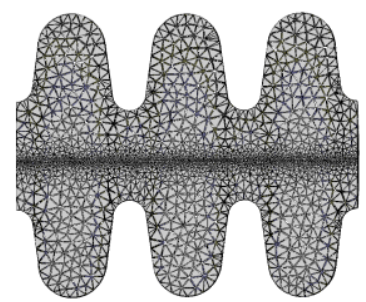

Figure 1: Final adaptive mesh produced using HFSS. A virtual box was used to create extra refinement around the wire.

order to adequately simulate the wire within the cavity it was necessary to create a "virtual object" in the vicinity of the wire to force additional mesh lines in this region. Half symmetry boundary conditions have been invoked in this simulation. The corresponding electric field for this configuration is illustrated in Fig 2. It is clear that the 
TEM mode, evident in the vicinity of the wire, couples to the transverse dipole deflecting mode. However, the coupling is not so strong as to cause a severe distortion in the dipole mode

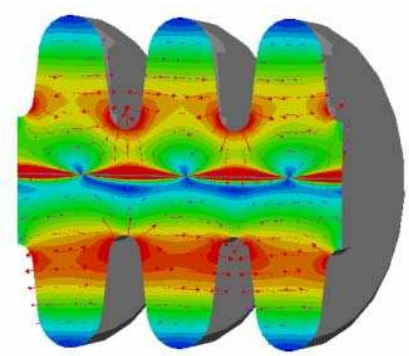

Figure 2: Electric field at a frequency of $3.9 \mathrm{GHz}$ for a 3cell cavity operating in a $\pi$ mode in which a wire is displaced $2 \mathrm{~mm}$ from the electrical centre

The scattering matrix component, $\mathrm{S}_{21}$, representing the transmission through this cavity is illustrated in Fig 3, along with an experimentally determined transmission normalised through the device under test (DUT) with respect to the reference vessel:

$$
\bar{S}_{21}=S_{21}^{D U T} / S_{21}^{\mathrm{Ref}}
$$

The impedance can be related to the scattering matrix by modelling the DUT with a transmission line loaded with a distributed series resistance [10-12]. Provided the distributed impedance makes a sufficiently small perturbation to the impedance reference vessel, there are several approximate formula that can be used depending on the frequency and length of the DUT [12].

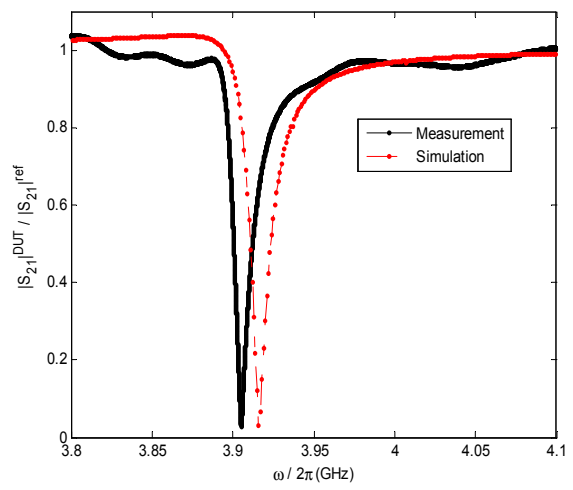

Figure 3: Measured and simulated $\bar{S}_{21}$ for a 3-cell cavity.

For cavities relatively long compared to the wavelength of the mode, the approximate "log" formula is adequate to represent the beam impedance:

$$
Z_{\|}=-2 Z_{C} \ln \bar{S}_{21}
$$

Here $Z_{c}$ is the impedance of a coaxial line with an inner radius corresponding to that of the radius of the wire, $\mathrm{r}_{\text {inner }}$, an outer radius given by that of the reference vessel, $r_{\text {outer }}$, and $Z_{0}$ the impedance of free space:

$$
Z_{C}=\frac{Z_{0}}{2 \pi} \ln \left(\frac{r_{\text {outer }}}{r_{\text {inner }}}\right)
$$

In the experiment the radius of the wire and reference vessel are $250 \mu \mathrm{m}$ and $18 \mathrm{~mm}$ respectively and this makes the impedance of the coaxial line $256 \Omega$. Thus, using Eq. 2 to calculate the impedance of the cavity results in the impedance curves illustrated in Fig 4.

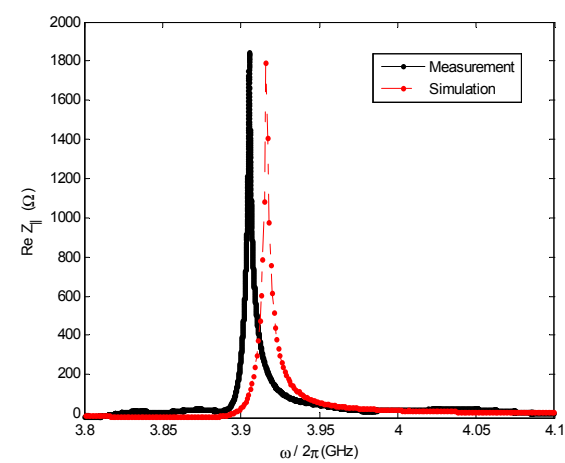

Figure 4: Impedance for the fundamental $3.9 \mathrm{GHz}$ dipole mode in a 3 -cell crab cavity loaded with a wire wire.

In order to compute the characteristic loss factor (the total energy lost by a point source transiting the cavity per unit charge squared [13]) we fit a Lorentzian function [14] to the impedance and obtain the data presented in Table 1.

Table 1: Frequency, loss factor and R/Q for a 3-cell crab cavity with a wire offset at $2 \mathrm{~mm}$.

\begin{tabular}{|l|l|l|l|}
\hline Parameter & $f(\mathrm{GHz})$ & $\begin{array}{l}k_{\text {loss }} \\
(\mathrm{V} / \mathrm{nC} / \mathrm{m})\end{array}$ & $\begin{array}{l}R / Q \\
\left(\Omega / \mathrm{cm}^{2}\right)\end{array}$ \\
\hline Measurement & 3.905 & 10.31 & 84.07 \\
\hline Simulation & 3.916 & 11.80 & 96.07 \\
\hline \% Error & 0.3 & 14.5 & 14.3 \\
\hline
\end{tabular}

The difference between the predicted and measured mode frequency is approximately $11 \mathrm{MHz}$, indicating that the technique is a reasonably accurate method of measuring the cavity frequency. The discrepancy in the predicted and measured loss factor is larger because of the uncertainty of the alignment of the wire. Angular misalignments in the wire can result in the wire coupling to unwanted modes and it can limit the coupling to the desired mode.

We are in the process of extending the experiments and simulations to additional modes within the cavity. Furthermore, in order to understand the distribution of the higher order modes excited in the cavity we have conducted an eigenmode simulation of a single cell subjected to infinitely periodic boundary conditions. The results of this study are presented in the next section.

\section{INFINITE PERIODIC STRUCTURES}

A dispersion diagram delineating the HOMs of the crab cavity both without a wire and perturbed by the displaced wire are displayed in Figs 5 and 6, respectively. In each instance the dashed line represents the velocity of light line. The points at which the light line intersects the curves correspond to synchronism with the beam and 
maximum interaction occurs at these locations. This series of curves was obtained using the efficient computer

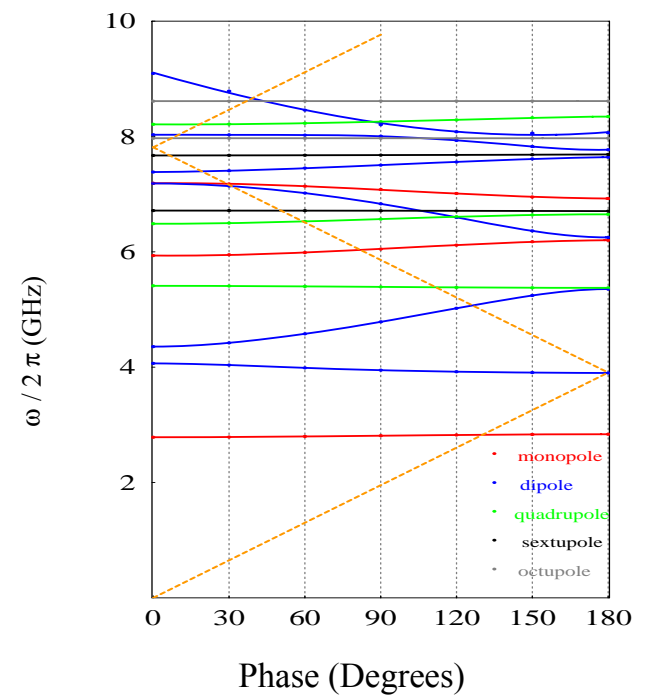

Figure 5: Brillioun diagram for unperturbed cavity

code GdfidL. It is notable that in the presence of the wire an additional mode is present, a TEM mode which couples to the monopole and dipole bands and this is illustrated in Fig 6. The coupling is largest in the region of $\pi$ phase advance per cell. This is evident as the TEM mode and monopole modes are markedly distorted in this

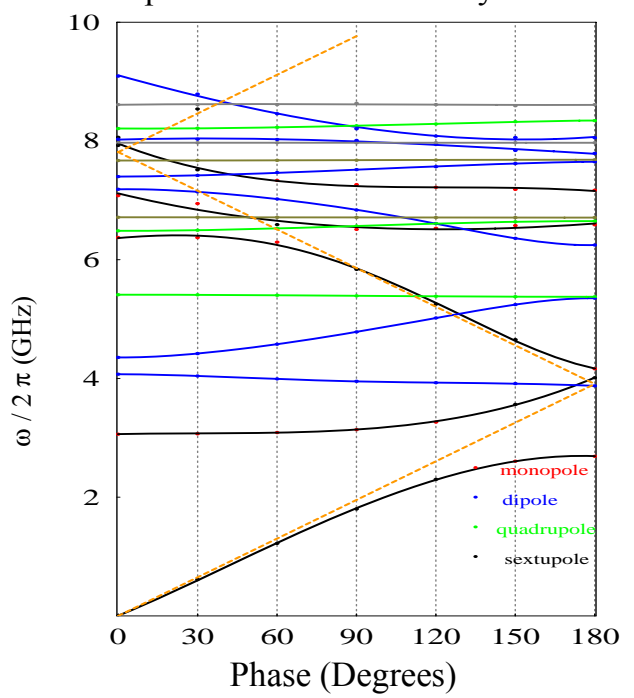

Figure 6: Brillioun diagram for a crab cavity perturbed by the presence of a wire offset $2 \mathrm{~mm}$ from the centre

region. The coupling to the dipole mode is observed more clearly by investigating field plots. An initial investigation of the alignment was conducted by tracking the position of a resonant peak as the wire offset is varied. The displaced wire couples to the dipole mode. From Slater's perturbation theorem [15] we predict a quadratic dependence on the frequency shift with wire offset. The result of monitoring the behaviour of the position of a dipole mode resonance with wire offset are presented in Fig. 7, along with a quadratic fit to the experimental data.

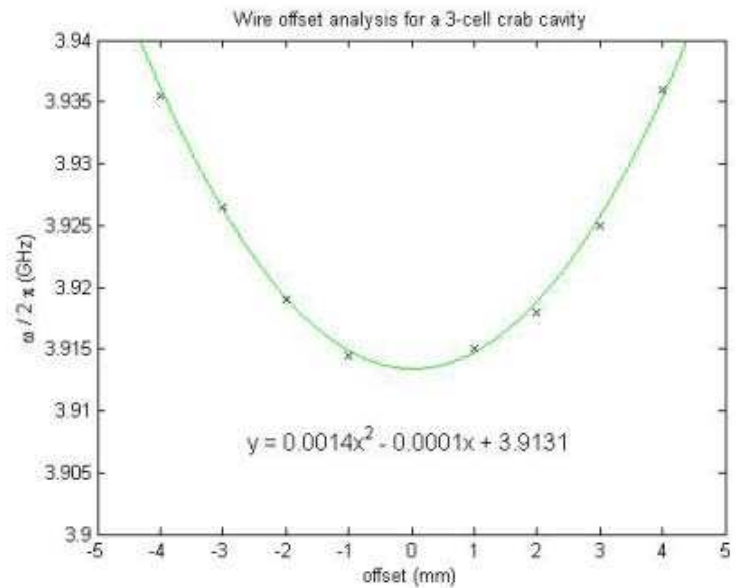

Figure 7: Frequency shift in the location of a dipole resonance versus wire offset

\section{DISCUSSION}

A comparison between measurement and simulation for the impedance, synchronous frequency and loss factor has indicated they are in reasonable agreement to within the expected experimental errors associated with this setup. Future work will expand the study to additional higher order modes. In particular the modes with the largest loss factors will be carefully measured and compared with theoretical predictions. An alignment study will be conducted based on the distribution of HOMs.

\section{REFERENCES}

[1] G. Burt et al. EUROTeV-Report-2006-098, 2006.

[2] M. McAshen, R. Wanzenberg. FERMILAB-TM2144, May 2001.

[3] G. Burt et. al. EUROTeV-Report-2006-058, 2006.

[4] F. Caspers, CERN-PS/RF/Note 2000-004, 2000.

[5] M. Sands and J.R. Rees, PEP-0095, 1974.

[6] N. Baboi et al. SLAC-PUB 10685, 2004.

[7] P. Goudket, G. Burt, R.M. Jones, These proceedings WEPMN077.

[8] HFSS. www.ansoft.com/hfss.

[9] W. Bruns, Proc. of PAC97, TET note 97/07, ww.gdfidl.de.

[10] V.G. Vaccaro. INFN/TC-94/023, 1994.

[11] L.S. Walling et al. Nucl. Instrum. Methods Phys. Res., A281, 433, 1989.

[12]E. Jensen, PS/RF/Note 2000-001, 2000.

[13] P.B.Wilson,. SLAC-PUB-4547, 1989.

[14] R.M. Jones et al., SLAC-PUB-10682, 2004.

[15] J.C. Slater. Microwave Electronics, New York: Van Nostrand, p. 81, 1950. 